\title{
Development of High-Resolution Transmission Electron Microscopes for Analysis of Biomolecular Structure
}

\author{
S. Motoki ${ }^{1}$, T. Kaneko ${ }^{1}$, H. Iijima ${ }^{1}$, Y. Shimizu ${ }^{1}$, I. Ishikawa ${ }^{1}$ and Y. Ohkura ${ }^{1}$ \\ 1. JEOL Ltd., 3-1-2 Musashino, Akishima, Tokyo 196-8558, JAPAN
}

Determining the structure of biological macromolecules, such as proteins and nucleic acids, is important for understanding their function. This leads to a mechanistic understanding of biological systems, and suggests pathways for discovery of therapeutic drugs. Structural analysis of biological macromolecules was first demonstrated in 1968 by DeRosier and Klug, with their three-dimensional reconstruction of negatively stained bacteriophage T4 [1]. Since liquid water is not compatible with the vacuum of an electron microscope, yet water retention is essential to maintain high-resolution molecular structure, methods of freezing the water in a vitreous state were eventually developed. This enabled the molecules to be imaged in a near-native state in the electron microscope. Yet, the high-resolution structure of these samples is easily compromised by electron-beam irradiation. As expected, lowering the temperature of the sample in the electron microscope was shown to reduce radiation sensitivity, and the lowest temperatures provided the most protection [2]. Thus, an electron microscope with a cryostage to maintain the low temperature, and a cryo-transfer system to avoid frosting or devitrification during specimen insertion, was called for.

In 1983, development of a dedicated cryo-transmission electron microscope (cryo-TEM) was started jointly by Y. Fujiyoshi and JEOL. Membrane proteins were chosen as the first specimens to be imaged, since they play important functions in the cell for trans-membrane transport and signaling, and knowledge of the structure and function of membrane proteins at the molecular level may clarify disease mechanisms. The three-dimensional structure of macromolecules can be obtained by several methods, including electron tomography, single-particle analysis, and electron diffraction. We chose electron diffraction, optimized for cryo-TEM. Either x-ray or electron diffraction can yield atomic or nearatomic results. Previously, x-ray diffraction was favored for obtaining the best resolution, but a threedimensional crystal is required. Electron diffraction is carried out with two-dimensional crystals, which are often easier to produce from biological macromolecules. By embedding two-dimensional crystals in lipid layers, followed by vitreous freezing, we were able to use electron diffraction to study membrane proteins at near-atomic resolution in a near-native state.

We used a liquid-helium reservoir centrally located in the TEM column to cool the specimen stage. The helium reservoir was surrounded by a liquid-nitrogen tank to suppress thermal radiation from the surrounding room environment. In order to avoid vibration from bubbling of the liquid helium, we made use of super-fluidity to cool the specimen, by which we could reach a temperature close to $2 \mathrm{~K}$. In keeping with best practices at the time, we employed a top-entry stage design for optimum stability. After much trial-and-error, the first-generation cryo-TEM was completed in 1986, which enabled highresolution imaging at $4.2 \mathrm{~K}$. Since then, the cryo-TEM has been improved, for example with the addition of an automatic specimen loader and an in-column Omega-type energy filter. The fifth-generation cryoTEM had been developed by 2005 . These instruments have made significant contributions to structural biology, for example clarification of the structure and function of aquaporin-1 [3] and the acetylcholine receptor [4]. 
The development of cryo-electron tomography in the 2000s, and its application to the structure of biological macromolecules, led to exciting results [5]. To meet the demands of electron tomography, tilt capability was added to the top-entry specimen stage of the sixth- and seventh-generation cryo-TEMs. In 2003, we developed the JEM-3200FSC, which has liquid-helium cooling and a side-entry specimenstage design.

With the advent of direct-electron-detection cameras, which offer improved low-dose imaging and which facilitate compensation for specimen drift and beam-induced motion, single-particle reconstruction now yields maps with resolutions below $3 \AA$ [6]. Recognizing that efficient automated data collection is essential for this work, we have implemented JDAS [7] on the current-generation cryoTEM. The current "CEYO ARM" is based on the JEM-ARM200F, which was designed for subAngstrom resolution. The microscope has a cold field-emission gun for the optimum coherency and image contrast, and the robust sample-loading system can select among twelve pre-loaded cryo samples.

References:

[1] D. DeRosier \& A. Klug, Nature 217 (1968), p. 130 - p. 134.

[2] Y. Fujiyoshi, Adv. Biophys. 35 (1998), p. 25-p. 80.

[3] K. Murata et al, Nature 407 (2000), p. 599-p. 605.

[4] K. Mitsuoka et al, J. Struct. Biol. 128 (1999), p. 34-p. 43.

[5] O. Medalia et al, Science 298 (2002), p. 1209-p. 1213.

[6] A. Bartesaghi et al, Science 348 (2015), p. 1147-p. 1151.

[7] Y. Aoyama et al, Microsc. Microanal, 12 (2014) p. 1114-p. 1115.

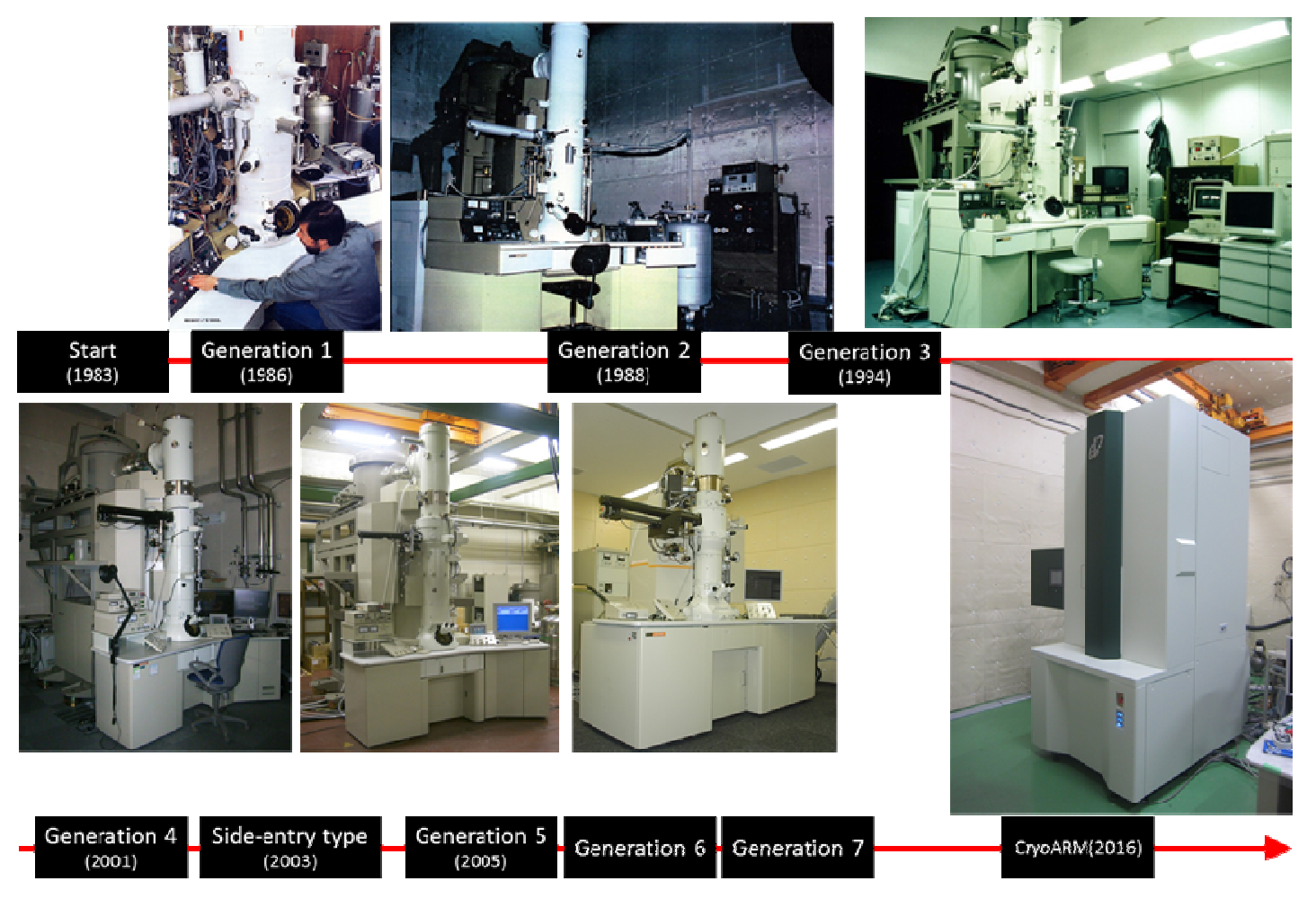

Figure 1. Timeline of cryo-TEM development. 